\title{
Socio-demographic Factors in Dysthymia and D[C]D
}

\author{
Megha Rathi ${ }^{1}$, Dr. Bidita Bhattacharya ${ }^{2}$ \\ ${ }^{1}$ M.Phil., Clinical Psychologist, Caring Minds, Kolkata, Mail id - megha_aims@yahoo.in \\ ${ }^{2}$ M. Phil., Ph.D, Associate Professor in Clinical Psychology, Institute of Psychiatry, Kolkata \\ Mail id - bidita.bhattacharya@gmail.com
}

\section{ABSTRACT}

Context : Social factors related to an individual play a key role in the development of any mental illness. Dysthymia and Dissociative [Conversion] Disorder are two important psychiatric disorders precipitated by stressful social factors and life events. Prior research has indicated significant stressors associated with both the disorders, but, however, there is a lack of comparative studies assessing the two disorders.

Aims : This study aims to explore the significant differences in socio-demographic factors among adults with Dysthymia, Dissociative [Conversion] Disorder and a group matched control group.

Setting and design : The comparative study included 60 individuals aged 18-45 years. 20 individuals had Dysthymia, 20 individuals had Dissociative [Conversion] Disorder and 20 individuals were belonging to normal control group. Semi structured clinical data sheet was administered to each subject to elicit the social factors. Hamilton Rating Scale for Depression, General Health Questionnaire and M.I.N.I International Neuro-Psychiatric Interview was used as a screening tool. All subjects were included in the study after obtaining their consent.

Statistics : Chi-Square was done to see significant difference in socio-demographic variables. Result \& Discussion : Results indicated significant difference within the three groups in terms of area, family type and marital status. Majority of patients with Conversion disorder belonged to rural area, were married and had extended families. Whereas, patients with Dysthymia mostly belonged to urban area, were unmarried or divorced and belonged to nuclear family.

Conclusion : The results of the study are significant and can be used for planning intervention programs for both the groups.

Keywords : Dysthymia, Dissociative [Conversion] Disorder, Socio-demographic factors

\section{INTRODUCTION}

Neurosis is a class of mental disorders that includes stress as one of its important precipitating factor. It is simply defined as a lack of ability to adapt to an individual's environment, difficulty to change one's

\section{Corresponding Author}

Dr. Bidita Bhattacharya

Financial support and sponsor : Nil

Conflict of Interest : There is no conflict of interest life patterns, and incapacity to develop a richer, complex and a satisfying personality pattern. ${ }^{[1]}$ Neurosis is thus a relatively mild mental disorder, characterized by anxiety, depression, dissociative or obsessive behaviour. In the present study, comparison has been made between Dysthymia and Dissociative [Conversion] Disorder, both of which are part of Neurotic Spectrum Disorder. 
Dysthymic Disorder refers to long standing and fluctuating low mood experienced as a part of the habitual self and represented through accentuation of traits observed in the depressive temperament. The essential features of dysthymic disorder include habitual gloom, brooding, lack of joy in life, preoccupation with inadequacy, feelings of guilt, irritability and anger and withdrawal from society. Dysthymia is a highly prevalent mental disorder with its rate varying from 3 to $6 \%$. It is estimated that approximately $7 \%$ of the primary care and up to $36 \%$ of the psychiatric outpatients have this disorder. ${ }^{[2]}$

Conversion Disorder is characterized by symptoms or deficits that affect voluntary motor or sensory functions which suggest a medical condition without any evidenced physiological cause. Thus, it is caused as a consequence of stressful or conflict ridden psychological factors. Lifetime prevalence of conversion symptoms varies from $25-30 \%$, whereas prevalence among general hospital inpatients needing psychiatric consultation has been reported between 5 to $16 \% .{ }^{[3]}$ With relation to sociodemographic features of conversion disorder, studies reveal that majority of the patients are young, female, single, unemployed, less educated, belong to urban population and have a prominent stress before the onset of symptoms. ${ }^{[4]}$

Studies have indicated that both the disorders are precipitated by a chronic stressor perceived by an individual. In both the disorders there are interpersonal disturbances identified as stressors leading to perceived deprivation of love, affection and care. In both the disorders, there is a need for narcissistic gratification. When this need is thwarted by real or perceived loss, patients with Dysthymia tend to introject the loss object and turn their anger on it leading to depressive symptoms; whereas patients with dissociative conversion disorders tend to repress such needs.) Thus, patients with Dysthymia use introjection (immature defense) whereas patients with Dissociative [Conversion] Disorder use repression and dissociation (neurotic defense) as a defense mechanism. ${ }^{[5]}$ Conversion
Disorder has been associated with depression; however, the present study attempts to see separate underlying factors for both the disorders. ${ }^{[6]}$

Socio-demographic factors influence the routine activities and tends to play an important role in the development and maintenance of both the psychiatric disorders under study. Worldwide, mental health conditions are reported to depend on relevant social and demographic factors. ${ }^{[7]}$ In the current study, relevant socio-demographic factors, namely, area, socio-economic status, marital status, religion, education and family type has been studied.

According to the previous researches, conversion disorder is more common in people with lower education level and its incidence decrease with increasing level of education. ${ }^{[8]}$ Further, married Indian population is more prone to conversion, and attacksarecommonduring premaritalengagements ${ }^{[9]}$ Conversion disorder tends to be temporally related to difficulties with primary support group including family circumstances. ${ }^{[10]}$ There are not many Indian studies, exploring the socio-demographic factors associated with Dysthymia. However, depression tends to be high in individuals with low income, lowered education and unsuccessful marriages. ${ }^{[1]}$

In terms of socio-demographic variables, subjects from 18 to 45 years of age were included in the study with minimum of primary level of education. In terms of age, participants above 45 years of age were not included as in females; it's an age for menopause which accounts for hormonal changes and mood fluctuations. This may cause additional stress in females, and that may have interfered with the results of the study. All the scales administered to the patients were of verbal nature and some degree of educational background is required to comprehend the items.

A study examined the relationship between social support, stressful life events and psychosocial dysfunction in a group of neurotic patients, which included patients of Generalized Anxiety Disorder, Dysthymia and Dissociative [Conversion] Disorder. 
Socio-demographic Factors in Dysthymia and D[C]D

Results indicated that Dysthymics perceive themselves to have significantly less social support in comparison to anxiety or dissociative disorder patients. Significant correlations between social support and life events and social support and dysfunction were also obtained. Ithas been observed that real or perceived lack of social support is negatively correlated with greater life stresses and increased occurrence of mental illness. ${ }^{[12]}$

This study purports to detect socio-demographic correlates in patients with Dysthymia and Dissociative [Conversion] Disorder so as to have a better insight about the social and environmental context of the mental illness.

\section{METHOD}

\section{PARTICIPANTS}

Sixty individuals from in and around Kolkata belonging to the age group of 18-45 years with mean age of 29 years for female and 30 for males participated in the study. Individuals with minimum of primary level of education were included in the study. The study comprises of two clinical groups, individuals with Dysthymia and Dissociative [Conversion] disorder, with 20 participants in each group. A normal comparative group (N-20) was taken with no psychopathology to better understand the factors associated with each disorder. Equal number of males and females were included in each group. For clinical groups, individuals meeting criteria for F34.1 Dysthymia or F44 Dissociative [Conversion] Disorder according to ICD 10 Diagnostic Criteria for Research (DCR), diagnosed by trained mental health professionals were selected from the Outpatient Psychiatry Department of Government Multi specialty hospital in Kolkata. On the selected participants, M.I.N.I and Hamilton Rating scale of depression was administered. Individuals who scored less than 7 for Dissociative Conversion Disorder and 12 for Dysthymia in the test were included in the study. For control group, the subjects were collected from the community from similar demographic characteristics with the clinical group.
These individuals were administered GHQ-12 and those scoring less than 2 were retained in the study. Participants presented with any chronic physical or mental illness were excluded from the study.

\section{ASSESSMENTS}

Consent form was prepared to seek consent from the targeted selected sample for their participation in the present study.

1. Sociodemographic data sheet: For the present study, an information schedule was prepared to elicit information on name, age, sex, address, maritalstatus, religion, occupation, qualification, personal and family income and family type along with current chief complaints of the client and brief history of their psychiatric illness.

2. General Health Questionnaire 12 : [13] It is developed by Goldberg in 1972 was used to assess psychological distress. GHQ-12 consists of 12 best items of the original 60 item questionnaire. GHQ is a self-administered tool, with scoring ranging from 0-1 for each item. Individuals scoring above 2 are addressed as psychologically distressed. The reliability for the scale is found out to be .90 from Likert method.

3. The Hamilton rating scale for depression : ${ }^{[14]}$ This is a 17 item rating scale to assess the presence and severity of depressive symptoms in the client. Inter-rater reliability for HAM-D total scores is found to be between $.80-0.98$ and the Test-retest reliability for the HAM-D has been reported to be as high as 0.81 . Validity of the HAM-D is found to range from 0.65 to 0.90 with global measures of depression severity.

4. The M.I.N.I. International Neuropsychiatric Interview 5: ${ }^{[15]}$ This is a brief structured interview for the major Axis I psychiatric disorders in DSM-IV and ICD-10. Validation and reliability studies have been done to compare the M.I.N.I. to the SCID-P and the CIDI. The results of these studies show that the M.I.N.I. has high validity and reliability scores and can be administered in a brief period of time. The modules I, J and 
$\mathrm{K}$ will be administered in the current study to rule out substance abuse and psychosis in the subject.

5. Kuppuswamy's Socio-economic status scale : ${ }^{[16]}$ This scale is based on a composite score considering the education and occupation of the head of the family along with monthly income of the family, which yields a score of 3-29. This scale classifies the study participants into high middle and low socio-economic status.

\section{PROCEDURE}

The prospective clinical patients for the study were contacted with the help of Out-patient Psychiatry department of a Government Multispecialty Hospital in Kolkata. The non-clinical comparative group participants were collected from the community keeping in mind the similarity in demographic characteristics with the clinical group in terms of age and gender. All participants were screened through clinical interview and assessment tools. Those who gave voluntary consent were taken. Along with the information schedule, all the scales were administered to the participants individually. Socioeconomic status for the participants were assessed using Kuppuswamy's Socio-economic Status Scale. The data collected was then scored and tabulated for further statistical treatment and determining the objectives.

\section{STATISTICAL ANALYSIS}

The data was statistically analyzed. The raw scores of the three groups were subjected to appropriate statistical analysis using the using Statistical Package for Social Sciences. Chi square was done in the current study to compare the three groups.

\section{RESULTS}

The current study is a cross sectional comparative hospital based study. The aim of the study is to see significant difference in three groups, i.e. Dysthymia, Dissociative [Conversion] Disorder and Normal control group with regards to categorical, socio-demographic variables like area, religion, family type, marital status, socio economic status and education.

Chi Square test is a non-parametric test which was used to compare between the three groups with regards to categorical, socio-demographic variables under study. The level $\mathrm{P}<0.05$ was considered as the cutoff value or significance. Thus, significance for all the result findings were interpreted at $95 \%$ confidence interval.

Results in Table 1 indicated significant difference within the three groups with respect to area, marital status and family type. There was no significant difference within the three groups with respect to socio-economic status, religion and education. From the table it can be seen that with respect to residence, the three groups differed with each other. Majority of Dissociative [Conversion] Disorder patients belong to rural background (60\%) whereas patients with Dysthymia belonged to urban background $(50 \%)$. With respect to marital status, majority of Dysthymia patients belonged to unmarried group $(60 \%)$ and majority of Dissociative [Conversion] disorder patients were married (46\%). With respect to family type, majority of Dysthymia patients belonged to nuclear family $(60 \%)$ and majority of Dissociative [Conversion] disorder patients belonged to extended family (50\%).

\section{DISCUSSION}

The present study is a cross sectional; hospital based comparative study conducted on individuals aged 18 to 45 years. The study comprises of two clinical groups, individuals with Dysthymia and Dissociative [Conversion] disorder and a normal control group. The current study aimed to compare the socio-demographic correlates between the patients with Dysthymia, Dissociative [Conversion] Disorder and non-psychiatric comparative group. Previous researches have shown that in both the disorders, there is mostly an on-going stressor that leads to maintenance of symptoms. Thus, in the current study, an attempt was made study the variables for both the disorder. 
In terms of socio-demographic variables, there was no significant difference in three groups with respect to socio-economic status, religion and education. There was a significant difference in three groups with respect to area, marital status and family type. With respect to area, it was observed that majority of patients with dissociative [conversion] disorder belonged to rural area. This may be due less availability of coping resources in rural areas to combat stress. Further, in rural area, the issues of survival and hardships have to be dealt with on daily basis and thus the coping resources are not sufficient to solve the daily problems. Conversion may provide an individual to escape from regular household and occupational chores and hassles leading to maintenance and reinforcement of symptoms. This is consistent with the findings which found that $80 \%$ of the patients with conversion disorder belonged to rural areas. ${ }^{[17]}$ The current study also indicated that majority of patients with Dysthymia belonged to urban areas. Urban Areas have high population densities are characterizedby higher rates of criminality, mortality, social isolation, air pollution and noise. ${ }^{[18]}$ As the extent of various social problems is related to urbanization, it may lead to more distress among individuals. Thus, significant difference in area can also be explained with reference to Drift hypothesis and Breeder hypothesis which was earlier linked to etiology of schizophrenia but now is also extended to the explanation of affective disorder and somatic symptoms. ${ }^{[19]}$ The drift hypothesis assumes on the one hand that sick and vulnerable people are more or less doomed to remain in socially unstable, deprived neighbour-hoods, while better on people move away. The second theory, the breeder hypothesis, assumes that various environmental factors cause illness. These can be physical factors (air pollution, small housing, and population density) and also social factors (stress, life events, perinatal aspects, social isolation). These factors are linked to urbanization.

In terms of marital status, it was indicated that majority of patients with conversion disorder were married. Marriage though is a positive event, it becomes stressful as it requires adjustment in the lifestyles of an individual and in interaction with the significant others in the environment. ${ }^{[20]}$ Further, marriage also leads to responsibilities that cause stress to both males and females leading to dissociating symptoms in order to escape stress. Moreover, individuals with Dysthymia were mostly unmarried. Thus, findings indicate that marriage is the central relationship for most adults and has beneficial effects for mental health. However, at the same time, troubled marriages have negative consequences on mental health. This finding can also be explained with Stress/social support hypothesis which states both positive and negative health correlates of marriage. ${ }^{[21]}$ This model sates that marriage causes stress in terms of increased responsibilities and sudden role changes but at the same time also increases positive social support.

In terms of family type, it was found that majority of patients with Dissociative [conversion] disorder patients belonged to extended family, whereas patients with Dysthymia belonged to nuclear or joint family. In extended family, there are more responsibilities and household chores, and equal importance is given to all members. In such situation, individual gets more attention when unwell leading to conversion symptoms (secondary gain). Moreover, in nuclear families, client feels lack of social support leading to more distress during stress leading to development of affective disorders. ${ }^{[22]}$

No study is free of limitation and the present study also has a few limitations. The study consisted of 60 individuals, 20 in each group. However, a larger samplecould haveyielded moreinformation, thereby enriching the study and increasing generalizability. The study entailed patient from only one hospital outpatient department, thus leading to problems in generalizability and external validity. Selfreport inventories were used in the study which automatically included the self-reporting biases along with the possibility of some faking. 


\section{CONCLUSION}

Dysthymia and Dissociative [Conversion] Disorder, both are major psychological disorders resulting from stress, and several studies have tried to address the stressor associated with each disorder. However, in view of dearth of early researches comparing the two disorders, the current study aimed to explore the differences in social factors among individuals with Dysthymia and Dissociative [Conversion] Disorder and a normal control group with no history of psychiatry. Studies showed significant differences in the social factors associated with both the disorders. Dysthymia and Dissociative [Conversion] Disorder are major psychiatric disorders developing rapidly in response to stressful life events. With the advancement of medicine, as the prevalence of disorder is increasing in psychiatric and medical set up, it is of paramount importance to better understand the problems plaguing them so that effective steps may be taken to deal with them.

\section{REFERENCES}

1. Boeree C.A Bio-Social Theory of Neurosis Retrieved. Br. J. Psychiatry 2002; 85 Supp 13 : 103-107.

2. Ellis H. Functional symptoms in neurology : Assessment. J Neurol Neurosurg Psychiatry 2002; 76 : 2-12.

3. Marsden CD. Hysteria : A neurologist's view. Psychol Med $1986 ; 16: 277-288$.

4. Bener A, Saad AG, Micallef R. Socio-demographic and Clinical Characteristics of patients with dissociative disorders in an Arabian Society. Med Princ Pract 2006; 15 Supp 15 : 362-67.

5. Sadock BJ, Sadock VA, Ruiz P. Kaplan \& Sadock's synopsis of psychiatry : Behavioral sciences/clinical psychiatry. 11thedn. Philadelphia : Wolters Kluwe. 2015.

6. Aamir S, Farooq S, Jahangir S. A comparison of life events in depressive illness and dissociative [conversion] disorders. J Pak PsychiatrSoc 2011; 8 Suppl 2 : 54-61.

7. Barua A, Ghosh MK, Kar N, Basili MA. Socio-demographic factors of geriatric depression. Indian J Psychol Med 2010; $32: 87-92$
8. Subramanian D, Subramanian K, Devaky MV, Verghese A. A clinical study of 226 Patients diagnosed as suffering from hysteria. Indian J Psychiatry 1980; 22 : 63-8

9. Pandit B, Kumar K, Yadav JS, Kaur S. Conversion Disorder: Soci0-demographic and Psychosocial correlates of patients presenting with Nonepileptic events. Delhi Psychiatry Journal 2011; 14 Suppl1 : 88-94

10. Voon V, Brezing C, Gallea C, Ameli R. Emotional stimuli and motor conversion disorder. Brain 2010; 133 Suppl5 : 1295-97

11. Udayar SE, Prasad DV. Epidemiological study of sociodemographic factors in relation to depression among the elderly people in a rural area of Chittoor district of Andhra Pradesh, India. Int J Community Med Public Health 2016, 3 Suppl1 : 161-165.

12. Kulhara, P, Chopra R. Social support, Social dysfunction and Stressful life events in Neurotic Patients. Indian J Psychiatry 1996; 38 suppl1 : 23-27

13. Goldberg DP. The detection of psychiatric illness by questionnaire. London : Oxford University Press. 1982.

14. Hamilton, M. A Rating Scale for Depression. J Neuro Psychiatry 1980; $23:$ 56-62.

15. Sheehan DV, Lecrubier $Y$, Sheehan KH, Amorim P, Janavs J, Weiller E, Hergueta T, Baker R, Dunbar GC. The MiniInternational Neuropsychiatric Interview (M.I.N.I.) : the development and validation of a structured diagnostic psychiatric interview for DSM-IV and ICD-10. J Clin Psychiatry 1998; 56 Suppl 20 : 22-33.

16. Kuppuswamy B. Manual of socioeconomic status (Urban). 1stedDelhi : Manasayan; 1981 : 66-72.

17. Maqsood N, Akram B, Ali W. Patients with conversion disorder, Psycho-social stressors and life events. Professional Medicine Journal 2010; 17 Suppl 4 : 715-720.

18. Freeman, H. Mental health and the environment, 1st edn London 1984 : Churchill Livingstone.

19. MaasJ, Verheij R. Green space, urbanity, and health : how strong is the relation? J Epidemiol. Community Health 2006; $60: 587-592$.

20. Irpati AS, Avasthi A, SharanP. Study of stress and vulnerability in patients with somatoform and dissociative disorders in a Psychiatric clinic in North India. Psychiatry Clin Neurosci 2006; 60 Suppl 5 : 570-574.

21. Burman B, MargolinG. (1992). Analysis of the association between marital relationships and health problems : An interactional perspective. Psychol Bull 1992; 112 : 39-63.

22. Kellar F, Ensminger T. Social Relationships and Mortality Risk : A Meta-Analytical Review. Am J Psychiatry 1997; 42Suppl5 : 80-94. 